\title{
Extramedullary tanycytic ependymoma of the lumbar spinal cord
}

\author{
Dong Ja Kim ${ }^{1}$, Man-Hoon Han ${ }^{2}$, SangHan Lee ${ }^{1}$ \\ ${ }^{1}$ Department of Forensic Medicine, School of Medicine, Kyungpook National University, Daegu, Korea \\ ${ }^{2}$ Department of Pathology, Kyungpook National University Hospital, Daegu, Korea
}

Received: September 24, 2019

Revised: October 29, 2019

Accepted: November 4, 2019

Corresponding author:

SangHan Lee

Department of Forensic Medicine,

School of Medicine, Kyungpook

National University, 130, Dongdeok-

ro, Jung-gu, Daegu 41944, Korea

Tel: $+82-53-420-4885$

Fax: +82-53-422-4712

E-mail: sanghan1@knu.ac.kr
Tanycytic ependymoma is a rare variant of ependymoma that commonly affects the cervical and thoracic spinal cord. It usually arises as intramedullary lesions, and extramedullary cases are extremely rare. We report a case of a 44-year-old woman who was diagnosed with tanycytic ependymoma in her lumbar spine at level 2-3. The tumor mass developed in an intradural extramedullary location. Histopathologically, tanycytic ependymoma can be misdiagnosed as schwannoma or pilocytic astrocytoma. Immunohistochemical findings such as strong positivity for glial fibrillary acidic protein, perinuclear dot-like positive patterns for epithelial membrane antigen, and focal positivity for S100 protein are helpful in diagnosing tanycytic ependymoma. It is important to be aware of this rare tumor to ensure appropriate patient management and accurate prognosis.

Keywords: Ependymoma; Glial fibrillary acidic protein; Lumbar vertebrae; Spinal cord neoplasms

\section{Introduction}

Tanycytic ependymoma is a histologically distinct rare subtype of ependymoma and is recognized as a grade II tumor in the latest World Health Organization classification in 2016 [1]. This tumor is usually found in the cervical and thoracic spine as an intramedullary mass but can also rarely present as an extramedullary mass in the lower spine [2]. Diagnosis in this location is difficult because the pathologic features resemble the findings of schwannoma and pilocytic astrocytoma. We present a case of tanycytic ependymoma in the lumbar spine and a brief literature review.

\section{Case}

All authors declare that written informed consent was obtained from the patient for publication of this case report and accompanying images.
A 44-year-old woman with complaints of a 4-year history of progressively worsening low back pain and sensory loss in her lower extremities visited our neurosurgery clinic. Four years prior, she had visited another hospital during which magnetic resonance imaging (MRI) revealed a 6-mm-sized intradural extramedullary mass in her lumbar spinal cord at level 2-3 (L2-3). The well-demarcated solid mass showed high signal intensity on T1-weighted imaging and low-to-intermediate signal intensity on T2-weighted imaging (Fig. 1). Surgery was recommended, but the patient refused. She had been intermittently treated with conservative therapy. However, recently, the pain in her low back, right buttock, and posterior aspect of her calf had aggravated. No muscle weakness or movement impairment was observed. Follow-up MRI showed that the mass had increased to $10 \mathrm{~mm}$ at L2-3. Her past medical history was unremarkable. The results of laboratory tests, including complete blood counts and liver and renal function tests, were within normal ranges. Under the preoperative diagno-

Copyright(C 2020 Yeungnam University College of Medicine

This is an Open Access article distributed under the terms of the Creative Commons Attribution Non-Commercial License (http://creativecommons.org/licenses/by-nc/4.0/) which permits unrestricted non-commercial use, distribution, and reproduction in any medium, provided the original work is properly cited. 

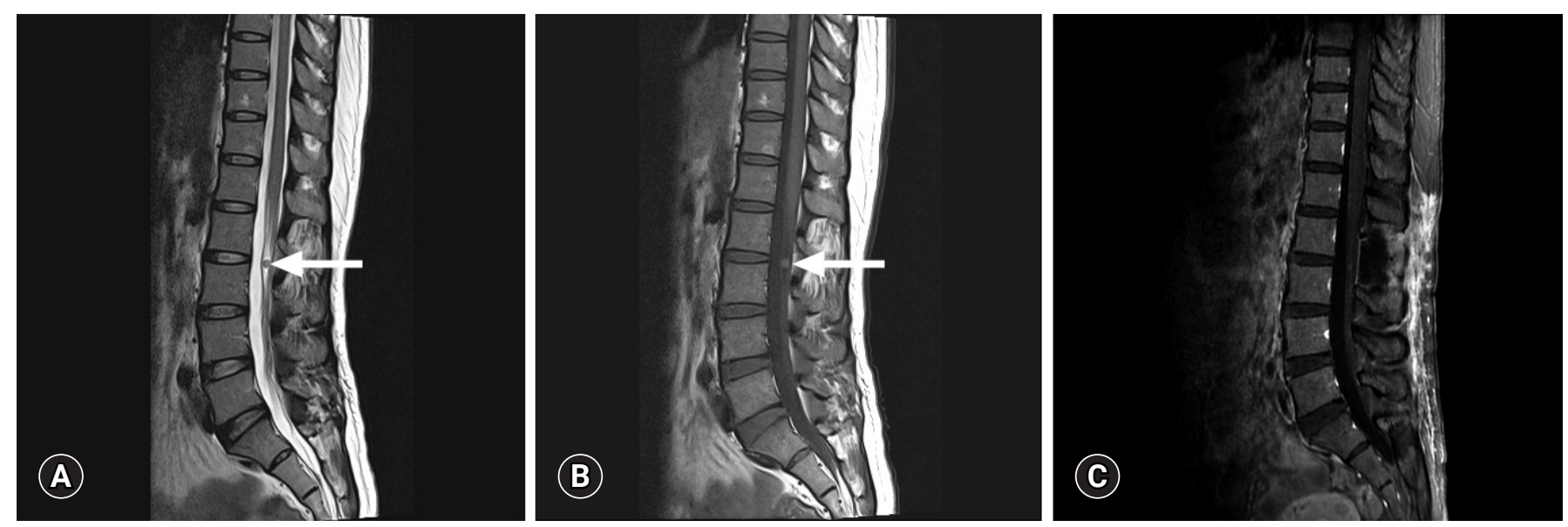

Fig. 1. Magnetic resonance imaging (MRI) findings. (A) Sagittal T2-weighted MR image of the lumbar spine showing a well-demarcated low-to-intermediate signal mass at lumbar level 2-3 spine (arrow). (B) Sagittal T1-weighted image showing high signal intensity (arrow). (C) No evidence of recurrence is visible in follow-up T1-weighted enhanced MRI.

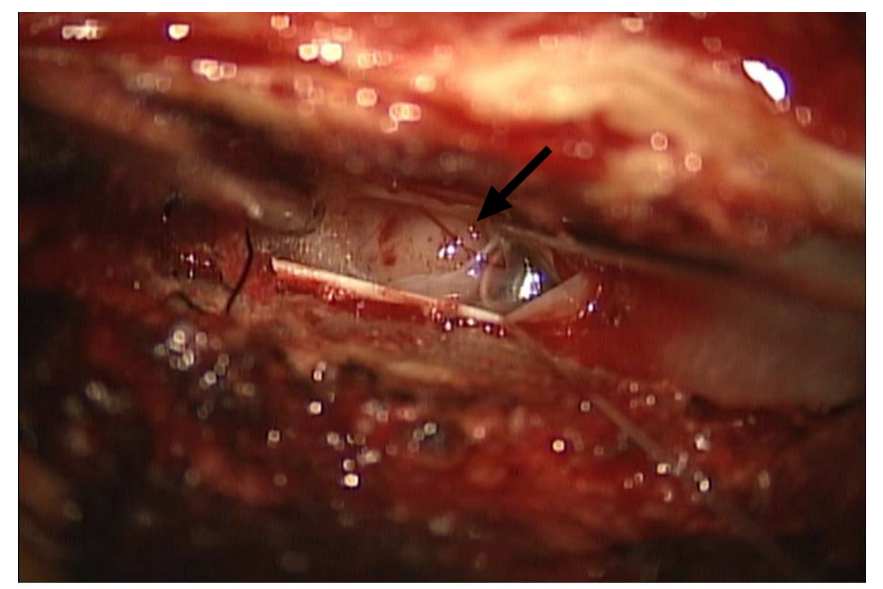

Fig. 2. Intraoperative findings. A well-demarcated tumor mass is visible upon opening of the dura (arrow). The nerve root is close to the mass but can be separated from the tumor surface.

sis of schwannoma, the patient underwent L2 hemilaminectomy. The opening of the dura revealed a well-demarcated tumor mass at the L2-3 spinal cord, which was not attached to the nerve root (Fig. 2). The gross total excision of the mass was performed.

Histopathologic analysis of the tumor mass revealed a non-encapsulated cellular neoplasm comprising short, vaguely intertwining fascicles of spindle cells. The tumor cells had oval or elongated nuclei with rich fibrillary processes resembling pilocytic astrocytoma. The nuclei displayed speckled chromatin and mitotic activity was inconspicuous. Hyalinized blood vessels and occasional perivascular pseudorosettes of elongated tumor cells were also observed (Fig. 3). However, true ependymal rosettes were absent. These histopathologic features resulted in a differential diagnosis including schwannoma, pilocytic astrocytoma, or other spindle cell neoplasm of the spinal cord. Immunohistochemical analyses, including those for glial fibrillary acidic protein (GFAP), vimentin, epithelial membrane antigen (EMA), S100 protein, CD34, and calretinin, showed tumor cells diffusely positive for GFAP and vimentin. S100 protein was focally positive, and EMA showed positive perinuclear dot-like or ring-like patterns consistent with ependymal differentiation (Fig. 4). The tumor cells were negative for CD34 and calretinin. Thus, a definitive diagnosis of tanycytic ependymoma was established. The postoperative results were uneventful, and the patient's symptoms improved.

\section{Discussion}

Ependymomas of the spinal cord usually arise within the cervicothoracic segment and are the most common intramedullary neoplasms of adulthood. The typical histopathologic features include a dense meshwork of fibrillary cytoplasmic processes forming perivascular pseudorosettes. Tanycytic ependymoma exhibits distinctive histologic features and was initially described by Friede and Pollak [3] in 1978. The term "tanycytic" refers to the spindle elongated cell morphology and the origin of the tumor cells from tanycytes, which are special and unique ependymal cells. Tanycytes are most commonly located in the wall of the third ventricle, in the circumventricular organs, and in the spinal cord [4]. In the spinal cord, they surround the spinal canal and radiate toward the grey matter. They are considered to participate in the communication between the cerebrospinal fluid, brain parenchyma, and vasculature [5].

Tanycytic ependymomas are commonly found in the cervical 


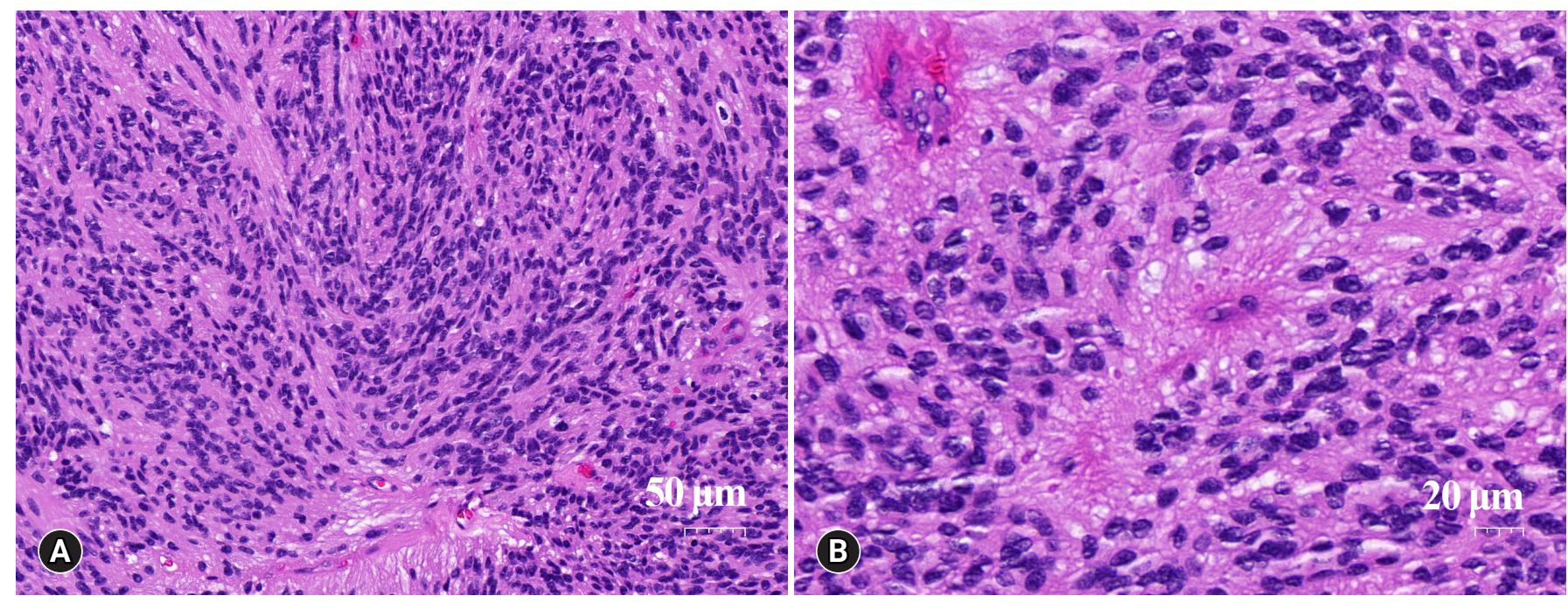

Fig. 3. Microscopic features. (A) Histopathologic analysis showing a moderately cellular neoplasm composed of short fascicles of elongated cells. The tumor cells have bland nuclei with no mitotic figures. The elongated cells are rich fibrillary processes (hemtoxylin and eosin stain, x200). (B) Hyalinized blood vessels and pseudorosettes are seen (hemtoxylin and eosin stain, x400).
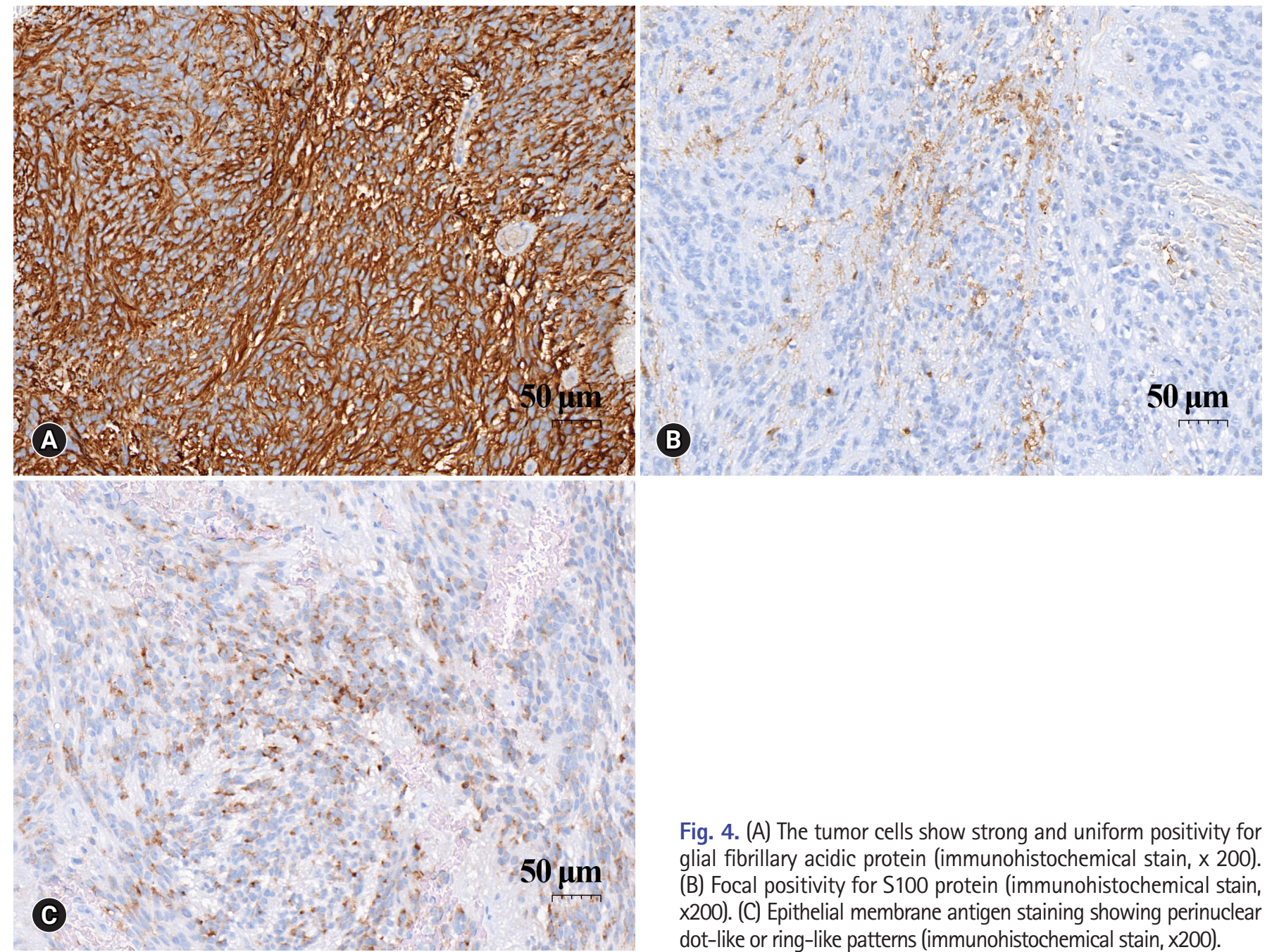

Fig. 4. (A) The tumor cells show strong and uniform positivity for glial fibrillary acidic protein (immunohistochemical stain, x 200). (B) Focal positivity for S100 protein (immunohistochemical stain, x200). (C) Epithelial membrane antigen staining showing perinuclear dot-like or ring-like patterns (immunohistochemical stain, X200). 
and thoracic spinal cord $[2,6]$. Tumors arising in the lumbar or thoracolumbar regions are very rare [2,4,7-9]. Extramedullary tanycytic ependymomas in the filum terminale are rarely reported [7-9]. According to the 40 cases of spinal cord tanycytic ependymoma reported by Tao et al. [2], only one and four cases were lumbar lesion and in extramedullary locations, respectively. Extramedullary tumors were found in the lower thoracic or lumbar spine. Tanycytic ependymoma accounted for approximately $1 \%$ of spinal cord tumors ( 40 of an estimated 4,000) and one patient had tumor recurrence after surgery. Thus, patients are usually expected to show long-term survival with a low rate of recurrence. Rare cases of tanycytic ependymoma associated with multiple endocrine neoplasia type 1 or neurofibromatosis type 2 have been also reported $[7,10,11]$. We found only one published case of tanycytic ependymoma in Korea in a Medline search of the English literature [10]. In that case, the patient had neurofibromatosis type 2 and was diagnosed with tanycytic ependymoma of the cervical spine.

The differential diagnosis on radiologic imaging features included schwannoma, neurofibroma, or myxopapillary ependymoma. In this case, the preoperative diagnosis was schwannoma. However, schwannoma may demonstrate more heterogeneous T2 signal hyperintensity. Most of the MRI features in previously reported cases of tanycytic ependymoma were T1-isointense and T2-hyperintense; however, the findings can be variable and non-specific. Therefore, the radiologic diagnosis of tanycytic ependymoma remains challenging. While tanycytic ependymoma is typically solid, cystic components were reported in half of the cases [12].

The histologic features show fascicles of spindle fibrillary tumor cells with low to moderate cellularity that can be misinterpreted as schwannoma, pilocytic astrocytoma, meningioma, or neurofibroma $[2,6,12,13]$. Myxopapillary ependymoma can be differentiated from tanycytic ependymoma because there are no pathological findings of a papillary arrangement of the tumor cells or deposition of basophilic mucinous materials. Immunohistochemical staining shows strong positivity for GFAP and focal reactivity for S100 protein in tanycytic ependymoma. The perinuclear dot-like or ring-like positive patterns of EMA are a peculiar feature in ependymoma. In contrast, schwannoma is negative for GFAP and EMA and uniformly positive for $\mathrm{S} 100$ protein. Moreover, schwannoma tends to be more cellular and has typical Antoni A and B patterns. Pilocytic astrocytoma can resemble tanycytic ependymoma. A pilocytic astrocytoma is strongly positive for GFAP but negative for vimentin. The findings of Rosenthal fibers and eosinophilic granular bodies are helpful in the diagnosis of pilocytic astrocytoma.

A case series of ependymoma reported that the Ki-67 labeling index appeared to be an important prognostic factor [14]. While
Ki-67 labeling index values of less than 4.0 have been associated with long survival times, a predictive threshold has not been established [15]. In our case, the Ki-67 labeling index was estimated to be less than $2 \%$. Radiotherapy can be considered for cases with incomplete resection and aggressive treatment such as chemotherapy is usually not indicated. There has been no evidence of tumor recurrence during the 1-year follow-up period after gross total resection (Fig. 1C).

The present case is a rare intradural extramedullary tanycytic ependymoma that developed in the L2-3 spine with slow tumor growth over 4 years. The histopathologic features were unique but accurate diagnosis was challenging and difficult due to unusual location and rarity. The precise diagnosis of tanycytic ependymoma is important because local recurrence is possible.

\section{Acknowledgments}

\section{Conflicts of interest}

No potential conflict of interest relevant to this article was reported.

\section{ORCID}

DongJa Kim, https://orcid.org/0000-0001-8462-3173

Man-Hoon Han, https://orcid.org/0000-0001-8856-553X

SangHan Lee, https://orcid.org/0000-0003-0390-3494

\section{References}

1. Louis DN, Perry A, Reifenberger G, von Deimling A, Figarella-Branger D, Cavenee WK, et al. The 2016 World Health Organization Classification of Tumors of the Central Nervous System: a summary. Acta Neuropathol 2016;131:803-20.

2. Tao X, Hou Z, Hao S, Zhang Q, Wu Z, Zhang J, et al. The clinical features and surgical outcomes of spinal cord tanycytic ependymomas: a report of 40 cases. World Neurosurg 2017;106:60-73.

3. Friede RL, Pollak A. The cytogenetic basis for classifying ependymomas. J Neuropathol Exp Neurol 1978;37:103-18.

4. Kawano N, Yagishita S, Oka H, Utsuki S, Kobayashi I, Suzuki S, et al. Spinal tanycytic ependymomas. Acta Neuropathol 2001; $101: 43-8$.

5. Dvoracek MA, Kirby PA. Intraoperative diagnosis of tanycytic ependymoma: pitfalls and differential diagnosis. Diagn Cytopathol 2001;24:289-92.

6. Krisht KM, Schmidt MH. Tanycytic ependymoma: a challenging histological diagnosis. Case Rep Neurol Med 2013;2013: 170791. 
7. Funayama T, Sakane M, Yoshizawa T, Takeuchi Y, Ochiai N. Tanycytic ependymoma of the filum terminale associated with multiple endocrine neoplasia type 1: first reported case. Spine J 2013;13:e49-54.

8. Radhakrishnan N, Nair NS, Hingwala DR, Kapilamoorthy TR, Radhakrishnan VV. Tanycytic ependymoma of filum terminale: a case report. Clin Neurol Neurosurg 2012;114:169-71.

9. D'Souza P, Martin WE, Bodhireddy S, Belirgen M. Extramedullary tanycytic ependymoma in a 12 -year-old boy. J Neurosci Rural Pract 2019;10:381-3.

10. Lim BS, Park SQ, Chang UK, Kim MS. Spinal cord tanycytic ependymoma associated with neurofibromatosis type 2. J Clin Neurosci 2010;17:922-4.

11. Tao XG, Hou ZG, Hao SY, Zhang JT, Liu BY. Two cases of spi- nal tanycytic ependymoma associated with neurofibromatosis type 2. Chin Med J (Engl) 2017;130:872-3.

12. Tomek M, Jayajothi A, Brandner S, Jaunmuktane Z, Lee CH, Davagnanam I. Imaging features of spinal tanycytic ependymoma. Neuroradiol J 2016;29:61-5.

13. Ortiz Ydel M, Perez Berenguer JL, Mercado Acosta J, Polo M, de Jesus-Garces O, Vega IE. Tanycytic ependymoma in a 76-yearold Puerto Rican male. Int J Clin Exp Pathol 2014;7:7789-94.

14. Wostrack M, Ringel F, Eicker SO, Jagersberg M, Schaller K, Kerschbaumer J, et al. Spinal ependymoma in adults: a multicenter investigation of surgical outcome and progression-free survival. J Neurosurg Spine 2018;28:654-62.

15. Prayson RA. Clinicopathologic study of 61 patients with ependymoma including MIB-1 immunohistochemistry. Ann Diagn Pathol 1999;3:11-8. 